\title{
A Convergent Continuous Multistep Process for the Preparation of $\mathrm{C}_{4}$-Oxime-Substituted Thiazoles
}

\author{
Edouard Godineau, ${ }^{*},+\| \odot$ Claudio Battilocchio, ${ }^{*}, \dagger, \ddagger, \|$ Matthias Lehmann, ${ }^{\dagger}$ Steven V. Ley, ${ }^{\ddagger}$ \\ Ricardo Labes, ${ }^{\ddagger}$ Letitia Birnoschi, ${ }^{\ddagger}$ Srinivas Subramanian, ${ }^{\S}$ C. S. Prasanna, ${ }^{\S}$ Amol Gorde, ${ }^{\S}$ \\ Mahesh Kalbagh, ${ }^{\S}$ Vivek Khade, ${ }^{\S}$ Anton Scherrer, ${ }^{\dagger}$ and Anthony C. O’Sullivan ${ }^{\dagger}$ (1) \\ ${ }^{\dagger}$ Syngenta Crop Protection, Process Research, Schaffhauserstrasse 101, CH-4332 Stein, Switzerland \\ ${ }^{\ddagger}$ Innovative Technology Centre, Department of Chemistry, University of Cambridge, Lensfield Road, Cambridge CB2 1EW, U.K. \\ ${ }^{\S}$ Syngenta Research and Technology Centre, Santa Monica Works, Corlim, Goa-403110, India
}

\section{Supporting Information}

ABSTRACT: We report a strategy designed for the rapid and convergent assembly of $\mathrm{C}_{4}$-oxime substituted thiazoles. Our approach relied on 3-bromo-2-oxopropanal $O$-methyl oxime 7 as a key building block. A three-step sequence to 7 was designed, which, for safety concerns, could only be operated in batch mode on limited scales $(\ll 100 \mathrm{~g})$. We describe herein how we addressed such a limitation, by designing a multistep continuous synthesis of this intermediate and further demonstrate the advantages of flow reactor configuration upon scaling up.

KEYWORDS: Bromination, continuous processing, hazardous chemistry, thiazole synthesis

$\mathrm{I}$ midazoles and thiazoles substituted with $N$-alkoxy oxime ethers moieties at $\mathrm{C}_{5}$ and $\mathrm{C}_{4}$, respectively, are generally regarded as useful features present in a number of biologically active substances (Figure 1).

For instance, compound $\mathbf{1}$ possesses interesting herbicidal properties. ${ }^{1}$ Similarly, the $\mathrm{C}_{4}$-alkoxyoxime-substituted thiazoles subclass recently gained interest. Compound 2, for example, expressed insecticidal activity, ${ }^{2}$ while substrates 3 and 4 have been associated with fungicidal activity. ${ }^{3-5}$ Heterocycles such as $\mathbf{3}$ are conventionally prepared using a linear sequence such as the one shown in Scheme 1.

Although this sequence works reasonably well, the preparation of analogs represented by the generic structure 6 is not very efficient and requires sequential synthesis steps. To streamline the preparation of such derivatives, we conceived an approach whereby thiourea 5 would be condensed directly with 3-bromo-2-oxopropanal $O$-methyl oxime 7 (Scheme 1). This approach is not only convergent but also pragmatic for the rapid and flexible production of thiazole-containing analogs. To satisfy the need for these exploratory programs, we required a robust access to oxime 7 , on a significant scale and quality.

The approach was based on the use of 2-oxapropanal oxime 9, as the primary building block, which can be prepared from three cheap feedstocks (Scheme 2); O-methylation of 9 would afford methoxyimino derivative 10, and a selective bromination of this intermediate would generate the final desired compound 7. It was soon evident that this sequence of chemical reactions would benefit from the implementation of a continuous flow process, due to both safety and handling of potentially hazardous intermediates (vide supra).

In this work, we disclose our efforts toward a robust and scalable preparation of intermediate 7 , and its later use in the convergent synthesis of the thiazole lead candidate 3 . We also describe the initial challenges, faced during the early batch scale-up campaigns, and how the use of continuous flow technologies has subsequently addressed the production of 7 , on a large laboratory scale.

Step 1: Synthesis of 2-Oxapropanal Oxime 9. Three different batch routes were investigated toward intermediate 9 (Scheme 3). A first approach started from pyruvaldehyde (Route A). A regioselectivity issue could be identified in the planning stage, since it is known that the aldehyde moiety is substantially hydrated in aqueous solution. ${ }^{6}$ This turned out to be the case; however, and we were unable to find conditions providing a fully selective access to intermediate 9, since byproducts 11 and 12 (see Scheme 4) were always competitively observed $(70: 30$ 9: $(11+12)$; see SI for further discussion). ${ }^{7}$ Given the difficulty in purging any of these undesired byproducts from 9, it was concluded that this approach was not optimal. Route B was also attractive, because of its concise nature and coming from commodity raw material. However, significant safety hurdles inhibited the delivery of 9 via this route in batch mode. ${ }^{8}$ Route $\mathrm{C}$ was therefore selected for our initial preparation of $\mathbf{9}$. Accordingly ethyl acetoacetate was nitrosylated, to give 8 (not isolated), which, upon treatment with sulfuric acid, a spontaneous release of $\mathrm{CO}_{2}$ and EtOH occurred to provide 9 in very good yield. ${ }^{9}$

Differential scanning calorimetry (DSC) analysis of intermediate 9 identifies exothermic activity between 140 and $275{ }^{\circ} \mathrm{C}$ with a heat output of ca. $2450 \mathrm{~J} / \mathrm{g}$, a value that represents $58 \%$ of the energy output of TNT. ${ }^{10,11}$ Under adiabatic conditions, isolated $\mathbf{9}$ has the potential to result in a temperature rise greater than $1400 \mathrm{~K}$. Route $\mathrm{C}$ enabled us to successfully prepare 9 in batch mode, provided the following

Received: March 29, 2018

Published: June 26, 2018 

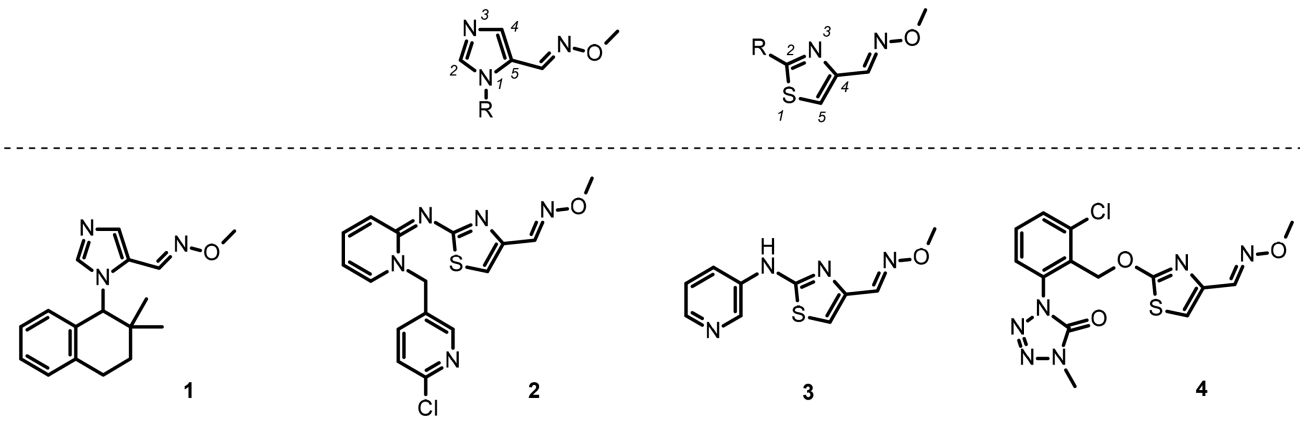

Figure 1. Biologically active imidazoles and thiazoles bearing a methoxy oxime moiety.

Scheme 1. Conventional and Convergent Approaches to Thiazoles Heterocycles Using 3-Bromo-2-oxopropanal O-Methyl Oxime 7, as New Intermediate of Synthesis

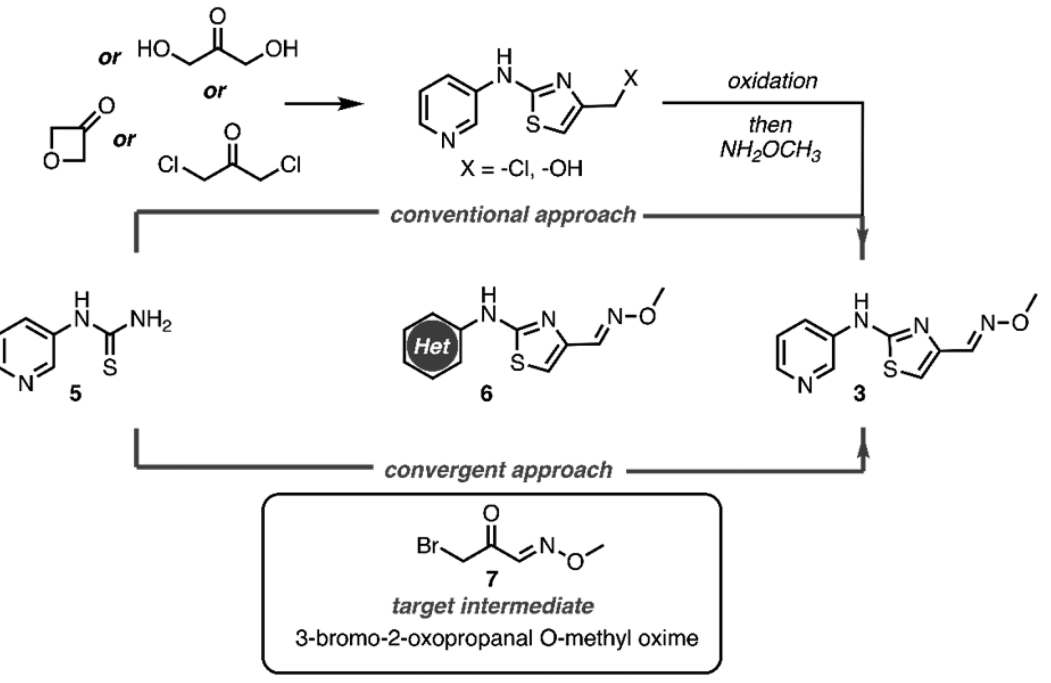

Scheme 2. Retrosynthetic Approach toward the Novel Synthesis of Pivotal Intermediate 7

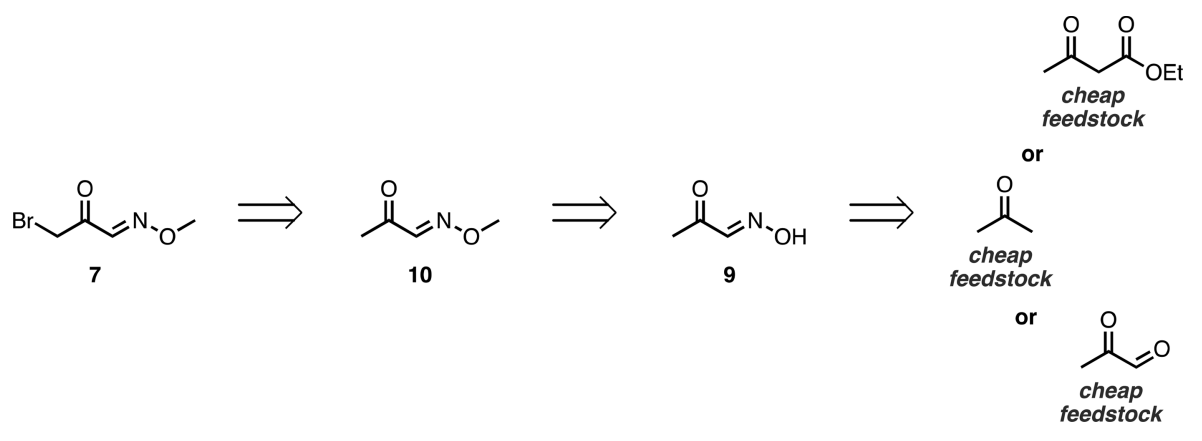

precautions were strictly followed: (i) ensure all handling of 9 is done at temperatures lower than $50-60{ }^{\circ} \mathrm{C}$; (ii) preferably use 9 in solution; and (iii) avoid any storage of 9, i.e. immediate consumption as such without any purification in the next stage. Although successful, we strongly recommend avoiding such a batch process on any scale and guide the reader toward the alternative described later where intermediate 9 is continuously generated and continuously consumed, therefore greatly mitigating the safety risks associated with its handling in a typical R\&D laboratory setup. In addition, we observed that intermediate 9 had the tendency to react, upon storage at rt (at least on small scale), to a mixture of oxime and bis-oxime byproducts 11 and 12 (Scheme 4a; see SI). For these reasons, we avoided further manipulations and purification of 9 and always used the material directly in the next steps. For further scale-up, a continuous flow process to prepare and consume 9consequently making the storage inventory of 9 as low as possible-would therefore represent an advantage in overcoming these significant stability and safety concerns.

Route $\mathrm{C}$ was not an ideal starting point for a continuous process since the reaction mixture appeared to gradually become triphasic owing to $\mathrm{CO}_{2}$ evolution (which would cause reactor pressurization) and precipitation of various solids. We foresaw these issues to be potentially problematic for the translation into a flow process. We therefore re-examined Route B. This alternative approach entailed the use of acetone reacting together with tert-butyl nitrite (Scheme 5). ${ }^{12}$ We could develop a continuous flow setup where tert-butyl nitrite and catalytic quantities of $\mathrm{HCl}$ are combined, leading to the in 
Scheme 3. Potential Approaches to the Synthesis of 2Oxopropanal Oxime 9

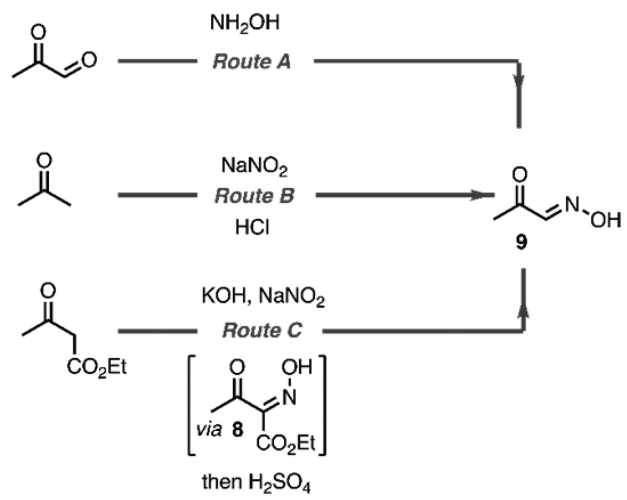

situ formation of nitrosyl chloride ${ }^{13}$ as a nitrosylating species. ${ }^{14}$ This intermediate reacts rapidly ${ }^{15}$ with acetone to generate the $\alpha$-nitrosylated acetone which then readily isomerizes to 9 . The reactor output was directed toward a Y-piece where it was then combined with a concentrated aqueous ammonia solution to quench the reaction.

Both mixing and temperature were found to be crucial parameters in order to produce high quality material, with the ideal temperature of the reactor being set at $10{ }^{\circ} \mathrm{C}$. A relative stoichiometry of 4.5:1.0:0.3 between acetone, tert-butyl nitrite, and $\mathrm{HCl}$ resulted in a robust protocol. Acetone was initially pumped at $2.00 \mathrm{~mL} / \mathrm{min}$ and combined at a T-piece with tertbutyl nitrite, delivered neat at $0.8 \mathrm{~mL} / \mathrm{min}$, and the mixture was combined with $\mathrm{HCl}$ (12 $\mathrm{M}$ aqueous solution, preloaded in a polymeric perfluoroalkoxy (PFA) loop and pumped at 0.16 $\mathrm{mL} / \mathrm{min}$ ) using an interdigital mixing unit. ${ }^{7}$ The reagents mixture was directed to a reactor coil (PFA, 1/16', $2 \mathrm{~mL}$ volume) kept at $10{ }^{\circ} \mathrm{C}$, and the output was then combined at a Y-piece with concentrated aqueous ammonia solution (14 M aqueous solution, preloaded in a PFA loop and pumped at 0.15 $\mathrm{mL} / \mathrm{min}$ ). The solution was collected in a flask cooled at 10 ${ }^{\circ} \mathrm{C}$. Analysis of the collected material for this initial run indicated intermediate 9 was obtained in 88\% yield (94\% purity). Under the conditions shown, the reaction productivity equated to $316 \mathrm{mmol} / \mathrm{h}$, namely $7.6 \mathrm{~mol} /$ day.

Step 2: Synthesis of 2-Oxopropanal O-Methyl Oxime 10. Intermediate 9 is easily converted to methoxyimino derivative $\mathbf{1 0}$ in good yield using dimethylsulfate in the presence of sodium hydroxide as the base. Careful reaction control was required since $\mathbf{1 0}$ is not stable under basic conditions. In addition, byproducts 13 and 14 are deemed to
Scheme 5. Flow Synthesis of Hydroximinoacetone 9

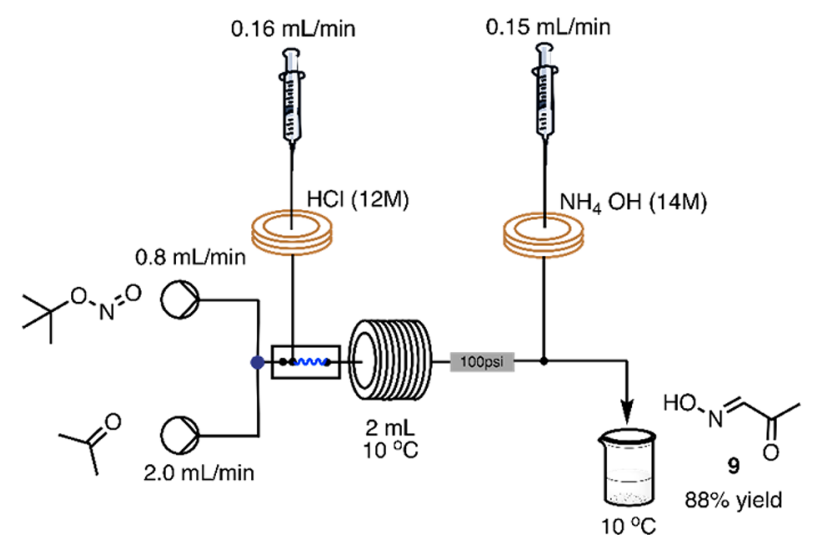

be equally unstable under basic conditions (Scheme 6). Obtaining $\mathbf{1 0}$ in a suitable quality was not a straightforward

Scheme 6. Synthesis of Intermediate 10 (from 9) and Byproducts 13 and 14

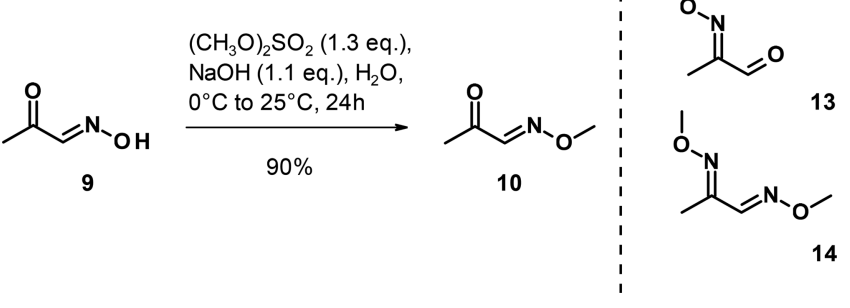

process and could only be realized by a multiple purification operation (see SI). Batch distillation in particular was found to be an unsuitable technique, as the residue remaining in the distillation vessel, after ca. $70 \%$ of the expected amount of $\mathbf{1 0}$ had been collected, was found to be thermally unstable. We tentatively attributed this exothermic decomposition to an accumulation of compound $\mathbf{1 4}$ in the residue remaining in the distillation vessel (see SI).

DSC analysis of intermediate $\mathbf{1 0}$ indicated slightly reduced safety concerns when compared to 9. The DSC analysis nevertheless pointed toward a series of consecutive and fairly complex exothermic events (at least 3 ), which are detected between $184{ }^{\circ} \mathrm{C}$ and ca. $345^{\circ} \mathrm{C}$. The measured heat release was ca. $1870 \mathrm{~J} / \mathrm{g}$. Under adiabatic conditions, this would result in a temperature rise greater than $1100 \mathrm{~K}$. Furthermore, the decomposition pattern of this compound clearly indicated the

Scheme 4. (a) Byproducts 11 and 12, Observed upon Storage of 9 and (b) Synthesis of 9 Using Ethyl Acetoacetate

a)

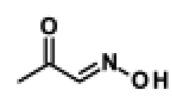

9

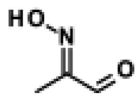

11

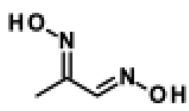

12

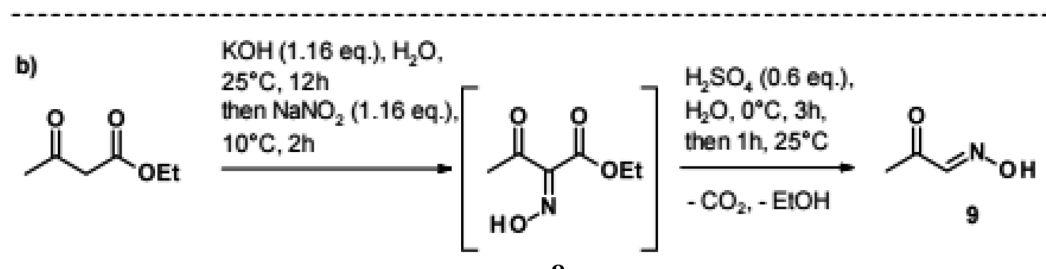

8 
Scheme 7. Reaction Setup for the Preparation of Intermediate 10

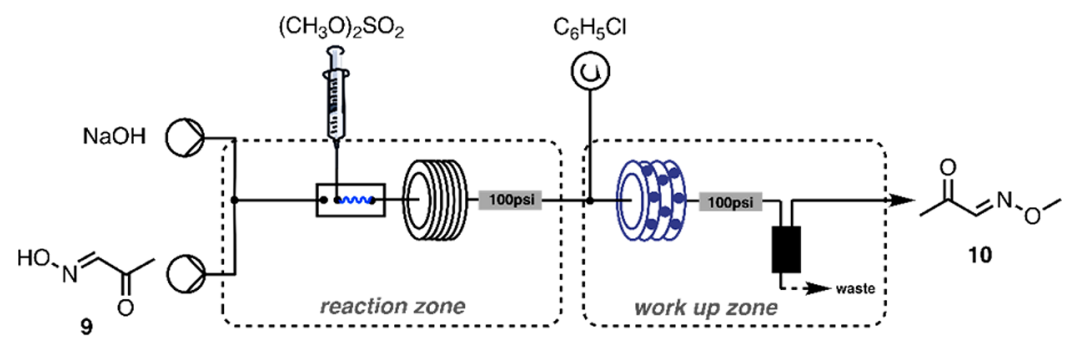

Scheme 8. Continuous Flow Synthesis of 10

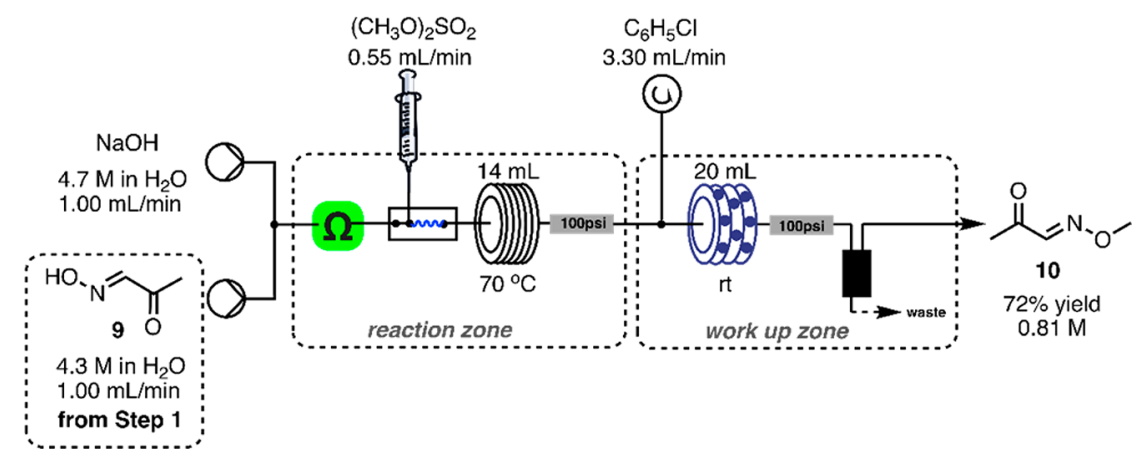

generation of gaseous byproducts. This set of information underlined, once again, the necessity of adopting continuous flow to mitigate the handling of these intermediates, especially on scale.

It was reasoned that implementing a suitable continuous flow reaction setup for this step would (a) more easily manage the safety risks; (b) improve selectivity and yield, by minimizing exposure of $\mathbf{1 0}$ to basic conditions and rapidly process the material in a telescoped fashion; and (c) avoid any purification stage.

Our initial flow screening was based on a system comprising 4 pumping units, a reaction zone, and a downstream zone (Scheme 7).

Systematic investigations (reagent relative stoichiometries, residence time, reactor temperature) quickly made apparent that the use of a diluted stream of dimethyl sulfate (up to 1.0 M) did not yield more than $7-10 \%$ of the desired product $\mathbf{1 0}$. This could arguably be attributed to an unfavorable mass transfer due to the biphasic nature of the reaction (Scheme 7). Indeed, it was noticed that increasing the concentration of the dimethyl sulfate feed significantly influenced the conversion to the desired product 10 (as detected by LC-MS), with yield being highest when using neat dimethyl sulfate. Notably, these changes did not affect the extraction process.

On the other hand, the use of a high concentration of the aqueous feedstocks of $\mathrm{NaOH}$ and 2-oxapropanal oxime 9 was found to be crucial for the optimal extraction of the product into the organic phase. The yield of extracted intermediate 10 increased from a $6 \%$ yield, using aqueous $2 \mathrm{M} \mathrm{NaOH}$ and $1 \mathrm{M}$ aqueous 9 , to a $38.5 \%$ yield when using aqueous $4 \mathrm{M} \mathrm{NaOH}$ and $3 \mathrm{M}$ aqueous 9. In all cases, the purity of resulting methoxyoxime $\mathbf{1 0}$ was found to be much improved over the batch process, further highlighting the benefits of the continuous approach over the batch process.

Our optimized protocol was as follows (Scheme 8): an aqueous solution of intermediate $9(4.3 \mathrm{M})$ and an aqueous solution of $\mathrm{NaOH}(4.7 \mathrm{M})$ were pumped at a $1.00 \mathrm{~mL} / \mathrm{min}$ flow rate for each channel and combined. The generated stream was passed through a conductivity cell, ${ }^{16}$ so as to monitor the steady state, next merged; with a dimethyl sulfate feed, and introduced neat and continuously at a flow rate of $0.55 \mathrm{~mL} / \mathrm{min}$. At such low flow rates $(2.0$ to $2.5 \mathrm{~mL} / \mathrm{min})$, efficient mixing is very difficult to reach. ${ }^{17}$ In order to achieve the best possible mixing of the biphasic mixture, we chose to use interdigital mixers ${ }^{18,19}$ which afforded as expected improved reliability when compared to simpler T-piece mixing units. The reaction mixture was reacted further in a $14 \mathrm{~mL}$ reactor coil (PFA, 1/16' o.d.) heated at $70^{\circ} \mathrm{C}$, before reaching a third mixing $\mathrm{T}$-piece, where chlorobenzene was combined with the reaction stream to perform an extraction step. Chlorobenzene was chosen as an extracting solvent as it is (a) easier and safer to handle on scale than $\mathrm{CH}_{2} \mathrm{Cl}_{2}{ }^{20}$ and (b) compatible with the subsequent electrophilic bromination step (vide infra, $\mathrm{CH}_{2} \mathrm{Cl}_{2}$ was used as a solvent in the bromination step). This downstream operation was aided by the use of a "static mixer coil". ${ }^{21}$ The output of the reaction system was next directed toward a liquid/liquid separator (for description, see SI). The collected organic phase was analyzed via ${ }^{1} \mathrm{H}$ NMR (against an internal standard, see SI) and indicated a $72.5 \%$ yield of intermediate $10(0.81 \mathrm{M}$ solution in $\mathrm{PhCl})$, with almost no detectable traces of any impurities.

Under the conditions described, the output of the reactor was calculated to be $187 \mathrm{mmol} / \mathrm{h}$ (equating to $4.49 \mathrm{~mol} /$ day).

Step 3: Synthesis of 3-Bromo-2-oxopropanal Omethyl Oxime (7). The bromination step to convert 10 into 7 was next investigated. This step proved to be very difficult to control and poorly reproducible during initial scaleup in batch mode. Selectivity in the bromination (mono- vs dibromination) was the main issue encountered. Methanol helped to improve the selectivity toward 7 , presumably via a selective in situ protection of compound 7 into its corresponding dimethoxyacetal. ${ }^{22}$ Compound 7 could be isolated after distillation under reduced pressure, on a $40 \mathrm{~g}$ scale in up to 58\% yield, as a 95:5 mixture together with 14 . 
Scheme 9. Continuous Flow Synthesis of 7

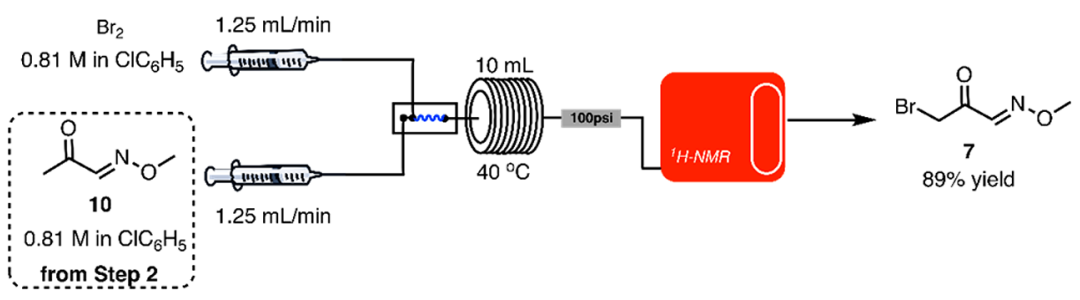

Scheme 10. (a) Continuous Flow Synthesis of 7, with Integrated Workup and (b) Using Neat Bromine

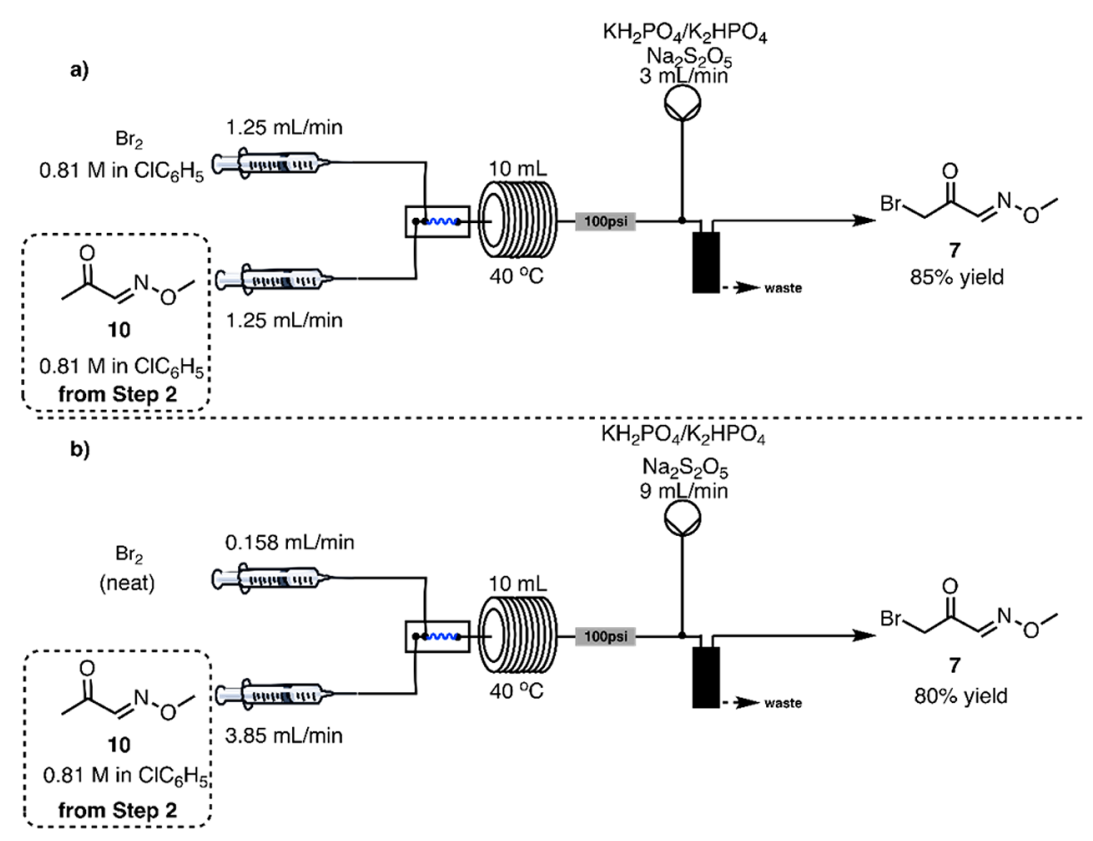

Methanol as an additive, while providing a selectivity benefit, introduced the undesired generation of gaseous $\mathrm{MeBr}$ as a byproduct. On larger scale, this technical drawback annihilated the selectivity benefit brought by the alcoholic additive. We did not investigate other heavier alcohols as additives. More importantly, we also observed a clear gradual erosion of the yield of the reaction on scaling up, such that on $>200$ g scales, the yield decreased to $25-35 \%$. We strongly suspected this lack of reproducibility on scale-up to be related to mass and/or heat transfer issues in batch mode. ${ }^{23}$

Safety evaluation for the intermediate 7 (see SI) indicated an onset decomposition temperature at $232{ }^{\circ} \mathrm{C}$, but with a slightly reduced heat release when compared to 10. Overall, DSC investigations indicate exothermic activity between $170^{\circ} \mathrm{C}$ and ca. $278{ }^{\circ} \mathrm{C}$ with a heat output of ca. $1350 \mathrm{~J} / \mathrm{g}$ which, ${ }^{11}$ under adiabatic conditions, 7 has the potential to cause a temperature rise greater than $800 \mathrm{~K}$; gas evolution was also observed on decomposition of 9 and 10. Finally, we observed gradual decomposition (color change and strength erosion on storage) of compound 7 over time, which led us to always use freshly generated material.

Altogether this batch process, although suitable for the first delivery of material, was therefore not satisfactory to sustainably provide 7 .

We recognized here another opportunity to take advantage of continuous technology to overcome these challenges. To achieve specific stoichiometry of reagents, we chose to use syringe pumps. Solutions of methoxyoxime 10 and bromine (both in chlorobenzene) were combined through an Innovia interdigital mixer. ${ }^{18}$ The reaction was then directed to a residence time coil, prior to analyzing the reaction output using an in-line NMR. ${ }^{24}$

A $0.81 \mathrm{M}$ solution of $\mathbf{1 0}$ in chlorobenzene (directly from step 2) was pumped at a flow rate of $1.25 \mathrm{~mL} / \mathrm{min}$ and combined with a $0.81 \mathrm{M}$ solution of $\mathrm{Br}_{2}$ pumped at $1.25 \mathrm{~mL} /$ min, before reacting in a PFA coil reactor, heated at $40{ }^{\circ} \mathrm{C}$, to provide 7 in $89 \%$ yield with just $2 \%$ of the dibrominated byproduct 15 also present (Scheme 9). This confirmed our hypotheses that improved mixing and heat transfer management would result in improved yield and selectivity for 7 . In order to refine our setup, we integrated a workup process to quench in-line any remaining $\mathrm{Br}_{2}$ and neutralize the acidity of the postreactor stream (Scheme 10). To this end, the reaction output was merged with an aqueous feed containing a phosphate buffer $(2 \mathrm{M})$ and sodium bisulfite $(0.5 \mathrm{M})$ (Scheme 10a). A standard workup of the reaction flow stream afforded 7 in $85 \%$ yield, although 7 was preferably generated on demand and directly telescoped into the next step as described below.

In order to increase the throughput of this step, we decided to use neat $\mathrm{Br}_{2}$ (Scheme 10b). Adjustment of the continuous flow parameters (reagent stoichiometry, feed concentrations, flow rates, reactor volume) led to an intensified process, with minimal configurational disruption of our previously designed platform. In particular, the solution of $10(0.81 \mathrm{M})$ was delivered at $3.85 \mathrm{~mL} / \mathrm{min}$ and combined using an interdigital mixer ${ }^{7}$ with neat $\mathrm{Br}_{2}$, delivered at $0.158 \mathrm{~mL} / \mathrm{min}$. The resulting stream was then sent into a $10 \mathrm{~mL}$ reactor coil (PFA, 1/16' o.d.) heated at $40{ }^{\circ} \mathrm{C}(2.5 \mathrm{~min}$ residence time $)$. The output 
Scheme 11. Flow Synthesis of the Thiazole Target, with Stage 4 (Synthesis of 5) and 5 (Synthesis of 3)

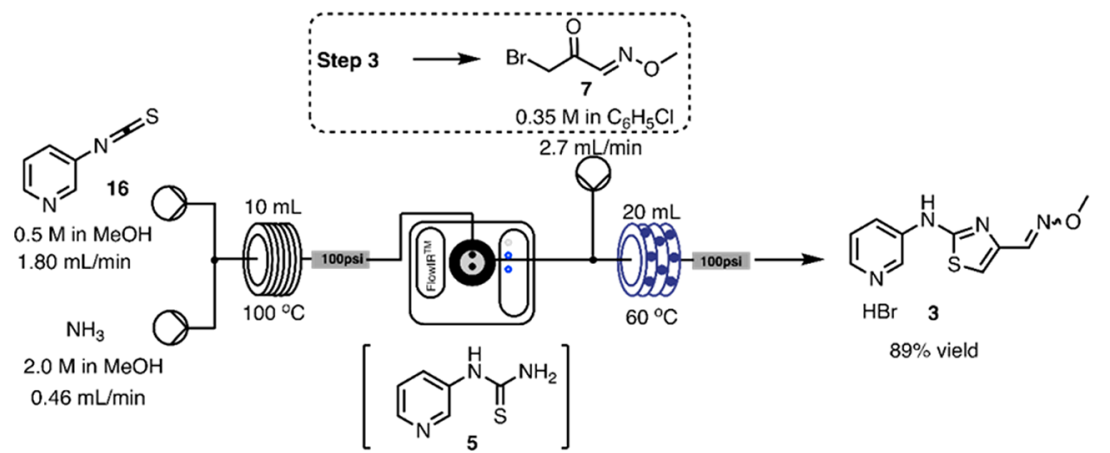

Scheme 12. Fully Telescoped Synthesis of the Thiazole Target 3

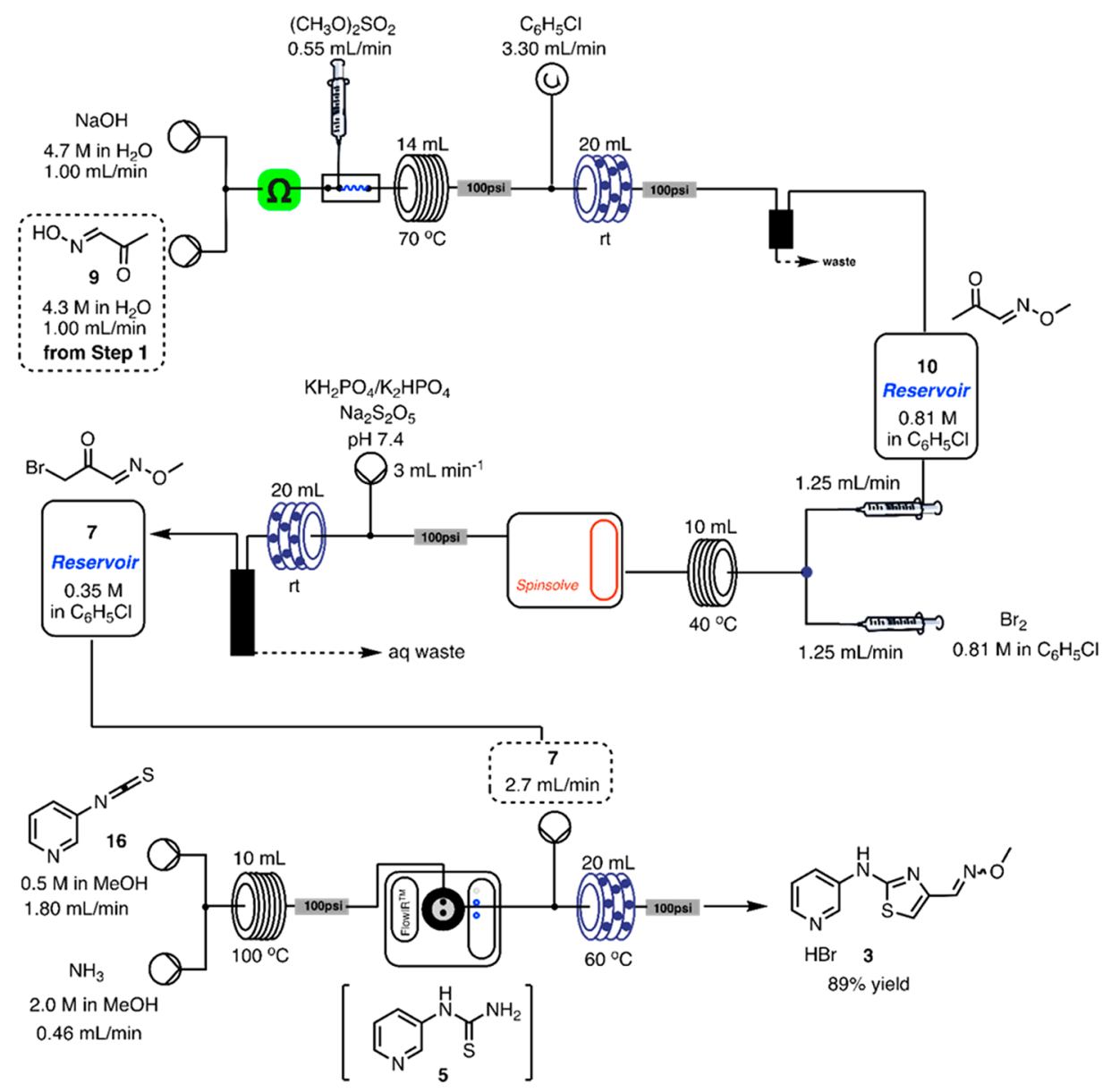

was quenched in-line using the same technique described above (aqueous quench delivered at $9.00 \mathrm{~mL} / \mathrm{min}$ ) and then analyzed. This afforded 7 in $80 \%$ yield, with excellent selectivity (20:1). Pleasingly, this intensified process produced an output of $149 \mathrm{mmol} / \mathrm{h}$ of intermediate $7(26.9 \mathrm{~g} / \mathrm{h})$, equating to $3.59 \mathrm{~mol} /$ day $(646 \mathrm{~g} /$ day $)$.

Step 4 and 5: Synthesis of 2-(Pyridin-3-ylamino)thiazole-4-carbaldehyde O-Methyl Oxime (3). As mentioned above, intermediate 7 cannot be stored for long period of time. We further demonstrated that 7 , generated on-demand in a continuous manner, can be immediately elaborated into the targeted thiazoles. Such generation and consumption on demand greatly mitigates any decomposition of 7 over time and avoids safety issues associated with the accumulation or storage of 7 . We demonstrated this conceptual approach by preparing, as a showcase example, thiazole 3 . In order to do so, we adopted a strategy whereby we would condense 7 and thiourea 5. We decided to prepare this latter compound $\mathbf{5}$, directly in continuous flow mode, by reacting isothiocyanate 16 and ammonia. Our group has previous experience with amination reactions of this type ${ }^{25}$ thus making the process straightforward in our hands.

In practice, a solution of isothiocyanate $16(0.5 \mathrm{M}$ in $\mathrm{MeOH})$ was combined with a concentrated solution of ammonia $(2 \mathrm{M}$ in $\mathrm{MeOH})$ and reacted in a $10 \mathrm{~mL}$ reactor coil (PFA, 1/16' o.d., residence time $4 \mathrm{~min} 40 \mathrm{~s}$ ), heated at 100 ${ }^{\circ} \mathrm{C}$, and then analyzed in-line with the use of an in-line IR system to ensure complete conversion of $\mathbf{1 6}$ to 5 . The reactor 
output was then directed to a T-piece where the thiourea intermediate 5 was combined with a stream of $7(0.35 \mathrm{M}$ in chlorobenzene, flow rate $2.7 \mathrm{~mL} / \mathrm{min}$ ). It was further reacted in a $20 \mathrm{~mL}$ reactor ${ }^{8}$ ( 4 min residence time), heated at $60{ }^{\circ} \mathrm{C}$, and the output collected to produce 3 in $89 \%$ yield, as a mixture of $E / Z$ isomers (Scheme 11).

The process provided 3 with a productivity of $48.6 \mathrm{mmol} / \mathrm{h}$ $(11.4 \mathrm{~g} / \mathrm{h})$, equating to $1.17 \mathrm{~mol} /$ day $(273 \mathrm{~g} / \mathrm{day})$ which represents a good level of productivity for further implementation of the process.

Finally, we further exemplify the potential streamlining of the whole sequence and demonstrated that each step could be telescoped into an effective sequence, as shown in Scheme 12. The setup was conveniently used as a machine-assisted production unit of thiazoles. It avoided any accumulation of unstable intermediates $\mathbf{1 0}$ and 7 and only used stable feedstocks (we have not included the continuous in-line formation of intermediate 9 from acetone described earlier and therefore used freshly prepared aqueous solutions of $\mathbf{9}$ for our experimental program).

In conclusion, we have reported a multistep continuous synthesis of a new brominated building block (7), which we have applied for the synthesis of thiazole 3 . The sequence shows the flexibility of continuous processing techniques when dealing with hazardous and unstable intermediates, mitigating the safety risks associated with these chemistries. In addition to the safety benefit, we were able to demonstrate significant improvements in the robustness of the overall sequence to access intermediate 7. Our continuous setup enabled the ondemand generation of 7 and further resulted into a more efficient and convenient preparation of libraries of thiazoles derivatives, when compared to the more conservative approach described in Scheme 1 (top). Further work is ongoing to define a complete telescoped sequence (steps 1, 2, and 3) for the synthesis, on scale, of such derivatives. We believe the success of such a scale-up will rely on such a fully telescoped process, where inventories of intermediates $9, \mathbf{1 1}$, and 7, at any given time during the process, are kept as low as possible so as to mitigate safety risks.

\section{ASSOCIATED CONTENT}

\section{S Supporting Information}

The Supporting Information is available free of charge on the ACS Publications website at DOI: 10.1021/acs.oprd.8b00095.

Compounds characterization and data (PDF)

\section{AUTHOR INFORMATION}

\section{Corresponding Authors}

*E-mail: edouard.godineau@syngenta.com.

*E-mail: claudio.battilocchio@syngenta.com.

\section{ORCID}

Edouard Godineau: 0000-0002-5958-4317

Steven V. Ley: 0000-0002-7816-0042

Ricardo Labes: 0000-0003-4001-0775

Anthony C. O’Sullivan: 0000-0003-3957-0492

\section{Author Contributions}

"E.G. and C.B. contributed equally.

\section{Notes}

The authors declare no competing financial interest.

\section{ACKNOWLEDGMENTS}

We would like to thank Ian Priestley for discussion concerning process safety. We would like to thank Syngenta Crop Protection and the EPSRC (S.V.L., Grant Nos. EP/ K009494/1, EP/M004120/1 and EP/K039520/1) for financial support.

\section{REFERENCES}

(1) De Bruyn, M. F. L.; Van Lommen, G. R. E.; Lutz, W. R. Preparation and testing of 1,5-disubstituted $1 \mathrm{H}$-imidazoles as herbicides. Patent EP289066A1, 1988.

(2) Von Deyn, W.; McLaughlin, M. J.; Gockel, B.; Koerber, K.; Dietz, J.; Pohlman, M.; Kojima, K. N-substituted imino heterocyclic compounds for combating invertebrate pests. WO2015124606A1, 2015.

(3) Cristau, P.; Rahn, N.; Tsuchiya, T.; Wachendorff-Neumann, U.; Voerste, A.; Benting, J. Thiazolyl oxime ethers and hydrazones as crop protection agents. Patent WO2010066353A1, 2010.

(4) Shioda, T.; Yoshimoto, Y. Preparation of phenyltetrazolinone derivatives as agrochemical fungicides. Patent WO2015060461A1, 2015.

(5) More elaborated oximes have been shown to possess kinase type activity as well: Zhao, X. Z.; Hymel, D.; Burke, T. R., Jr. Application of oxime-diversification to optimize ligand interactions within a cryptic pocket of the polo-like kinase 1 polo-box domain. Bioorg. Med. Chem. Lett. 2016, 26, 5009-5012.

(6) Chari, R. V. J.; Kozarich, J. W. Synthesis and properties of halohydroxyacetones and halomethylglyoxals. J. Org. Chem. 1982, 47, 2355-2358.

(7) We have investigated in-depth a shortcut for the sequence, with the use of pyruvaldehyde and $O$-methyl hydroxylamine. The various investigations, i.e. solvents, acid additives, stoichiometries, and temperatures, led us to the conclusion that it is not possible to achieve the desired selectivity toward 10:

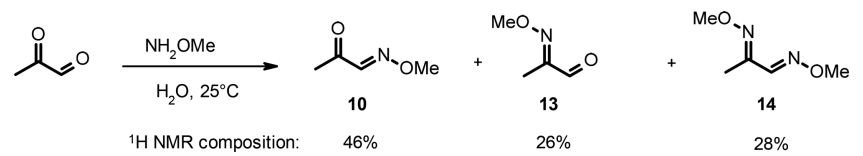

(8) Under oxidizing conditions acetone can lead to the formation of explosive triperoxides. Although detonation would only occur if such intermediates would crystallize out of solution, we anticipated severe restrictions about our ability to perform such an operation on large scale, under standard batch conditions. Consult for an examplehttp:// cenblog.org/the-safety-zone/2017/02/how-a-student-unintentionallymade-an-explosive-at-u-bristol/.

(9) (a) Freon, P. Preparation of $\alpha$-hydroxy aldehydes. Ann. Chim. Appl. 1939, 11, 453-518. (b) Zhang, C. Y.; Tour, J. M. Synthesis of Highly Functionalized Pyrazines by Ortho-Lithiation Reactions. Pyrazine Ladder Polymers. J. Am. Chem. Soc. 1999, 121, 87838790. (c) Sheremetev, A. B.; Shamshina, Y. L.; Dmitriev, D. E. Synthesis of 3-alkyl-4-aminofurazans. Russ. Chem. Bull. 2005, 54, $1032-1037$

(10) TNT has a heat release output of ca. $4180 \mathrm{~J} / \mathrm{g}$ : Appendix B8 Factors for Units Listed Alphabetically. In NIST SI Guide 2008.

(11) According to the UN transport regulations if a DSC shows for an organic molecule a heat output greater than $500 \mathrm{~J} / \mathrm{g}$ then it requires further testing to determine whether it has explosive properties.

(12) Leis, J. R.; Pena, M. E.; Williams, D. L. H. Involvement of the enol tautomers in the nitrosation of ketones. J. Chem. Soc., Chem. Commun. 1987, 2, 45-47. Coraor, G. R., Woodbury, N. J. Process For Preparing Oximinoacetone. Patent US2731499, 1956.

(13) Morton, J. R.; Wilcox, H. W. Nitrosyl Chloride. Inorganic Syntheses 2007, 4, 48-52.

(14) Nitrosyl chloride is known to be highly reactive: Beckham, L. J.; Fessler, W. A.; Kise, M. A. Nitrosyl Chloride. Chem. Rev. 1951, 48, 319-396. 
(15) Ridd, J. H. Diffusion Control and Pre-association in Nitrosation, Nitration and Halogenation. Adv. Phys. Org. Chem. 1978, 16, 1-49.

(16) The conductivity cell was positioned after the first reaction, and the output from the conductivity cell was used to determine steady state (see SI for more information). Conductivity cell: UniCond Sensor available from Mettler Toledo:https://www.mt.com/us/en/ home/products/Process-Analytics/conductivity-sensor/conductivityresistivity-cell-water/Unicond-2e.html.

(17) See also for micromixing devices characterization: Schwolow, S.; Hollmann, J.; Schenkel, B.; Röder, T. Application-Oriented Analysis of Mixing Performance in Microreactors. Org. Process Res. Dev. 2012, 16, 1513-1522.

(18) Slit interdigital micromixer SIMM-V2, Institut für Microtechnik, Mainz GmBH, Mainz, Germany. Channels $40 \mu \mathrm{m}, 200 \mu \mathrm{m}$ depth, internal volume, approximately $8 \mu \mathrm{L}$.

(19) MMH-040T interdigital mixer available from Invenios Europe $\mathrm{GmBH}$, now Corning.

(20) Prat, D.; Hayler, J.; Wells, A. A survey of solvent selection guides,. Green Chem. 2014, 16, 4546-4551.

(21) The static mixer coil available from Vapourtec: https://www. vapourtec.com/products/flow-reactors/large-diameter-tubularreactor-for-rapid-mixing-features/.

(22) Masilamani, D.; Rogic, M. M. Sulfuryl chloride as a reagent for selective chlorination of symmetrical ketones and phenols. J. Org. Chem. 1981, 46, 4486-4489.

(23) (a) Bourne, J. R.; Rys, P.; Suter, K. Mixing effects in the bromination of resorcin. Chem. Eng. Sci. 1977, 32 (7), 711-716.

(b) Bourne, J. R. Mixing and the Selectivity of Chemical Reactions. Org. Process Res. Dev. 2003, 7, 471-508.

(24) Magritek Spinsolve $43 \mathrm{MHz}$ was used: http://www.magritek. $\mathrm{com} /$.

(25) (a) Pastre, J. C.; Browne, D. L.; O’Brien, M.; Ley, S. V. Scaling Up of Continuous Flow Processes with Gases Using a Tube-in-Tube Reactor: Inline Titrations and Fanetizole Synthesis with Ammonia. Org. Process Res. Dev. 2013, 17, 1183-1191. (b) Browne, D. L.; O’Brien, M.; Koos, P.; Cranwell, P. B.; Polyzos, A.; Ley, S. V. Continuous-Flow Processing of Gaseous Ammonia Using a Teflon AF-2400 Tube-in-Tube Reactor: Synthesis of Thioureas and In-Line Titrations. Synlett 2012, 23, 1402-1406. (c) Cranwell, P. B.; O'Brien, M.; Browne, D. L.; Koos, P.; Polyzos, A.; Peña-López, M.; Ley, S. V. Flow synthesis using gaseous ammonia in a Teflon AF-2400 tube-intube reactor: Paal-Knorr pyrrole formation and gas concentration measurement by inline flow titration. Org. Biomol. Chem. 2012, 10, 5774-5779. 